\title{
Directional growth of polypyrrole and polythiophene wires
}

\author{
Prem S. Thapa, ${ }^{1, a)}$ Deok Jin Yu, ${ }^{1}$ James P. Wicksted, ${ }^{1}$ Jeffrey A. Hadwiger, ${ }^{2}$ \\ Joseph N. Barisci, ${ }^{3}$ Ray H. Baughman, ${ }^{3}$ and Bret N. Flanders ${ }^{1, a), b}$ \\ ${ }^{1}$ Department of Physics, Oklahoma State University, Stillwater, Oklahoma 74078, USA \\ ${ }^{2}$ Department of Microbiology and Molecular Genetics, Oklahoma State University, Stillwater, \\ Oklahoma 74078, USA \\ ${ }^{3}$ NanoTech Institute, The University of Texas at Dallas, Richardson, Texas 75083-0688, USA
}

(Received 29 September 2008; accepted 22 December 2008; published online 23 January 2009)

\begin{abstract}
This work establishes an innovative electrochemical approach to the template-free growth of conducting polypyrrole and polythiophene wires along predictable interelectrode paths up to $30 \mu \mathrm{m}$ in length. These wires have knobby structures with diameters as small as $98 \mathrm{~nm}$. The conductivity of the polypyrrole wires is $0.5 \pm 0.3 \mathrm{~S} \mathrm{~cm}^{-1}$; that of the polythiophene wires is $7.6 \pm 0.8 \mathrm{~S} \mathrm{~cm}^{-1}$. Controlling the growth path enables fabrication of electrode-wire-target assemblies where the target is a biological cell in the interelectrode gap. Such assemblies are of potential use in cell stimulation studies. (C) 2009 American Institute of Physics. [DOI: 10.1063/1.3072611]
\end{abstract}

Conducting polymer nanowires are a burgeoning subfield of the polymeric electronics arena. ${ }^{1}$ On a fundamental level, these wires serve as a testing ground for onedimensional transport theories that describe the Luttinger liquid $^{2}$ and Wigner crystal phases of electronic materials. ${ }^{3}$ In sensor applications, where analyte binding to porous wires modulates the resistance of electrode-wire-electrode assemblies, impressive sensitivities have been attained: submicrobar pressures of ammonia, nanomolar concentrations of DNA, and $\sim 10 \mathrm{fM}$ concentrations of micro-RNA (Ref. 5) have been detected with these devices. Polymeric wires are also promising electrophysiological materials. A recent study has demonstrated the electropolymerization of poly 3,4-ethylenedioxy-thiophene (PEDOT) filaments in neural tissue without compromising the viability of the surrounding neurons - a preliminary step toward remotely stimulating cells and tissue. ${ }^{6,7}$ A general requirement for all of these applications is the reproducible fabrication of electrode-wire-target assemblies, where the wire connects a user-selected electrode to a second electrode or a biological cell, enabling diagnostic instrumentation to be interfaced with the wire or target. Therefore, methodologies for the $d i$ rectional growth of polymeric wires are of significant interest.

Templated growth techniques are in wide use as they yield reproducible wire shapes and wire growth along predictable paths. To fabricate electrode-wire-electrode assemblies, the polymer may be electrodeposited in prefabricated channels that connect user-selected electrodes. ${ }^{8,9}$ Thus, directional growth is realized by engineering growth channels before electrochemically depositing the wire. Template-free approaches have been developed where the growth of polyaniline $^{4}$ and PEDOT (Ref. 10) nanowires occurs between the tips of two electrodes; the maximum wire length that was attained by these techniques was limited to $<10 \mu \mathrm{m}$. Dip-pen lithography relaxes the wire length constraint $^{11}$ but is restricted to applications where the use of a scanning probe is feasible. To broaden the range of situa-

\footnotetext{
${ }^{a}$ Present address: Department of Physics, Kansas State University, Manhattan, KS 66506-2601.

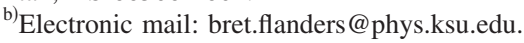

tions in which directional wire growth is possible, the present work establishes a simple approach to growing conducting polymer wires across distances as large as $30 \mu \mathrm{m}$ in on-chip circuitry to produce electrode-wire-target assemblies, where the target is either a second electrode or a biological cell.

To attain this goal, we have extended the directed electrochemical nanowire assembly (DENA) technique to the growth of polypyrrole (PPy) and PEDOT wires. This technique was previously used to grow metallic nanowires, ${ }^{12-14}$ where alternating voltages reduced and deposited metallic cations onto biased electrodes immersed in aqueous salt solutions. ${ }^{12}$ A similar approach is used here to electrochemically polymerize conducting polymer onto biased electrodes immersed in aqueous monomer solutions, enabling the growth of polymeric electrode-wire-target assemblies.

Figure 1(a) depicts the simple apparatus used to grow wires from aqueous pyrrole solution. The gap between the

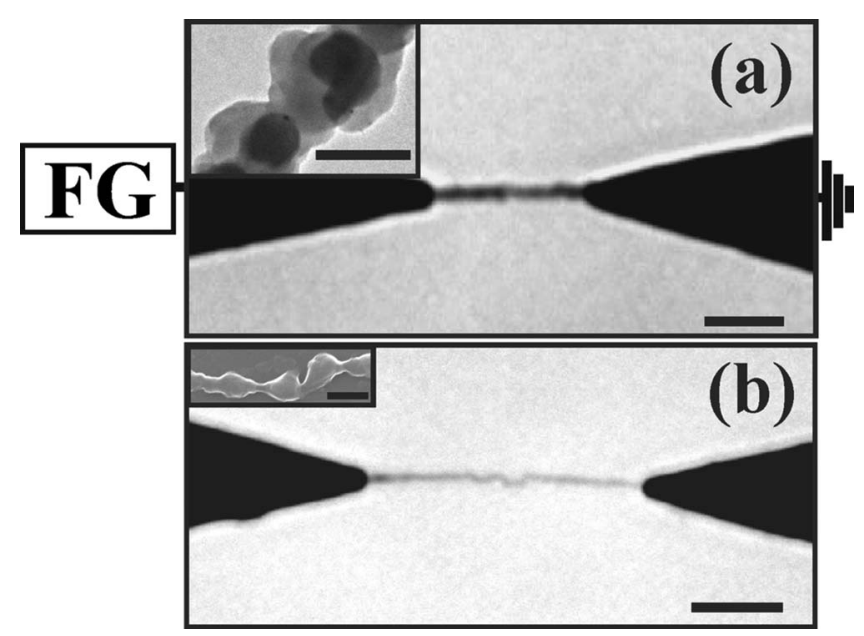

FIG. 1. (a) DENA apparatus for growing polymeric wires. FG designates a function generator. The optical micrograph shows a wire grown from aqueous pyrrole/SDS solution. Inset: a transmission electron micrograph of a PPy wire. (b) An optical micrograph of a wire grown from aqueous EDOT/ PSS solution. Inset: a scanning electron micrograph of a PEDOT nanowire. The scale bars represent $10 \mu \mathrm{m}$ except for those in the insets, which denote $500 \mathrm{~nm}$. 

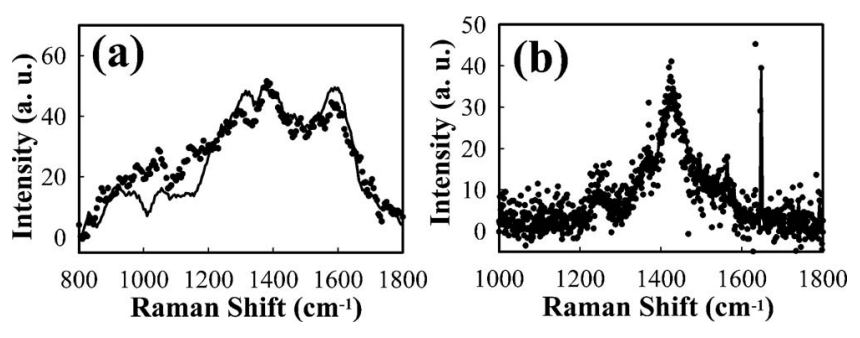

FIG. 2. (a) Micro-Raman spectra of (a) a wire grown from pyrrole solution (filled circles) and a PPy film (solid line) and (b) a wire grown from thiophene solution (filled circles) and a PEDOT film (solid line). a.u. is an abbreviation for arbitrary units.

opposing electrode tips is $18.8 \mu \mathrm{m}$. The electrodes were composed of $100 \mathrm{~nm}$ thick base layers of $\mathrm{Ti}$ and $500 \mathrm{~nm}$ thick top layers of $\mathrm{Au}$ that were deposited on fused silica substrates using standard lithographic techniques. ${ }^{15}$ A $10 \mu \mathrm{l}$ aliquot of aqueous solution containing $0.50 \mathrm{M}$ freshly distilled pyrrole and $0.50 M$ sodium dodecyl sulfate (SDS) was deposited across the electrodes. The pyrrole and SDS concentrations may be varied across the $0.05 M-0.50 M$ range, and $p$-toluene sulfonate may be used instead of SDS as the anionic dopant. To induce wire growth, a square-wave voltage signal $( \pm 3 \mathrm{~V}, 250 \mathrm{kHz})$ with a $50 \%$ duty cycle was applied to the left electrode, while the right electrode was grounded. Growth was stopped by turning off the voltage when the wire reached the opposing electrode. This process was observed on an inverted microscope (Leica, IRB). The resulting assembly is shown in Fig. 1(a). A transmission electron micrograph of a PPy wire segment is shown in the inset. The wire has a knobby structure with a lengthwise averaged diameter of $560 \mathrm{~nm}$.

Milder conditions were used to grow wires from aqueous 3,4-ethylenedioxy-thiophene (EDOT) solutions. The fiber in Fig. 1(b) was grown by depositing $10 \mu \mathrm{l}$ of aqueous solution containing $0.01 M$ EDOT and $0.02 M$ poly(sodium styrene sulfonate) (PSS) across the electrodes. Additionally, a smaller voltage-amplitude $( \pm 2.5 \mathrm{~V})$ and frequency $(100$ $\mathrm{kHz}$ ) were used to induce growth. The interelectrode gap in Fig. 1(b) is $30.2 \mu \mathrm{m}$. The inset depicts a scanning electron micrograph of the wire. Its structure is knobby, varying from 98 to $669 \mathrm{~nm}$ in thickness with a lengthwise averaged diameter of $340 \mathrm{~nm}$. A real-time movie of this wire growth process is available as supplementary material (see Ref. 16).

As Figs. 1(a) and 1(b) and the movie (Ref. 16) illustrate, the growth path is predictable: it follows the tip-to-tip line between the user-selected electrodes. This path is defined by the voltage profile in the solution. A full description of the underlying ionic diffusion and screening behavior lies beyond the scope of this letter.

The compositions of the DENA-grown wires were determined by micro-Raman spectroscopic measurements of the wire-laden electrode arrays. Wires grown from pyrrole solutions were characterized by illuminating the wire segments with $1.5 \mathrm{~mW}$ of $632.8 \mathrm{~nm}$ laser radiation, focused to a $2.0 \mu \mathrm{m}$ spot size; the monochromator entrance slit was $200 \mu \mathrm{m}$ wide. Figure 2(a) compares the wire spectrum (filled circles) to the spectrum of a PPy film (solid line) prepared by a standard protocol. ${ }^{17,18}$ Both spectra exhibit features that are characteristic of PPy: peaks at $1050 \mathrm{~cm}^{-1}$ (the symmetrical $\mathrm{CH}$-in-plane bending mode), at 1310 and $1375 \mathrm{~cm}^{-1}$ (the antisymmetrical $\mathrm{CH}$-in-plane bending

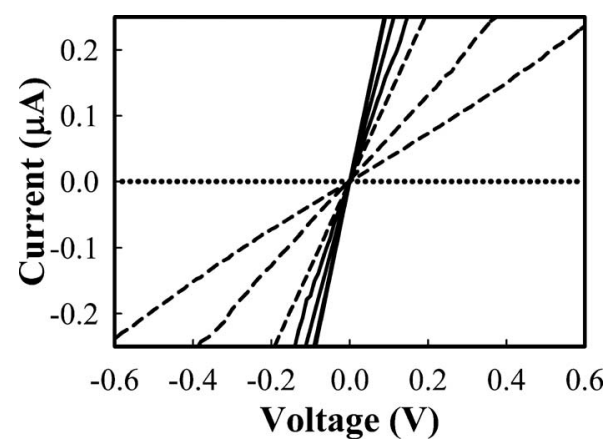

FIG. 3. $I-V$ profiles of electrode-wire-electrode assemblies with PPy wires (dashed lines), PEDOT wires (solid lines), and solution but no wire (dotted line)

mode), and at $1600 \mathrm{~cm}^{-1}$ (the $\mathrm{C}=\mathrm{C}$ stretching mode); ${ }^{18}$ hence, the DENA-grown wire is composed of PPy.

Figure 2(b) compares the micro-Raman spectrum of a wire grown from aqueous thiophene solution (filled circles) to the spectrum of a PEDOT film (solid line) that was prepared by a standard procedure. ${ }^{19,20}$ Both exhibit features at $1240 \mathrm{~cm}^{-1}$ (the $\mathrm{C}-\mathrm{C}$ stretching mode) and $1430 \mathrm{~cm}^{-1}$ (the symmetric $\mathrm{C}=\mathrm{C}$ stretching mode), indicating that this DENA-grown wire is composed of PEDOT.

To characterize the wire conductivities, we have measured current-voltage $(I-V)$ profiles of PPy and PEDOT electrode-wire-electrode assemblies. After wire growth, the assemblies were washed with pure water three times and allowed to dry before making two-point $I-V$ measurements with a source meter (Keithley, 2400). The profiles for three PPy wires (dashed lines) and six PEDOT wires (lines) are shown in Fig. 3. The resistances $R$ of the $\sim 16 \mu \mathrm{m}$ long PPy wires range from 0.76 to $2.49 \mathrm{M} \Omega$ (as given in Table I along with the individual wire lengths $l$ ). The resistances of the longer $(\sim 26 \mu \mathrm{m})$ PEDOT wires range from 0.35 to $0.49 \mathrm{M} \Omega$. We approximate the knobby wire shapes as uniform cylinders with diameters of $560 \pm 70 \mathrm{~nm}$ for PPy and of $340 \pm 70 \mathrm{~nm}$ for PEDOT; these are the lengthwise averaged diameter values obtained via electron microscopy. Using the relationship $\sigma=l / R A$ (where $A$ is the crosssectional area) and the values in Table I, the conductivities $\sigma$ of the individual PPy wires were calculated and reported in Table I. The average of these three determinations is $0.5 \pm 0.3 \mathrm{~S} \mathrm{~cm}^{-1}$; the uncertainty is the standard deviation of

TABLE I. Parameters for computing the polymeric wire conductivities.

\begin{tabular}{lcccc}
\hline \hline & $\begin{array}{c}\text { Length } \\
(\mu \mathrm{m}) \\
\pm 0.5 \mu \mathrm{m}\end{array}$ & $\begin{array}{c}\text { Diameter } \\
(\mathrm{nm}) \\
\pm 70 \mathrm{~nm}\end{array}$ & $\begin{array}{c}\text { Resistance } \\
(\mathrm{M} \Omega) \\
\pm 0.01 \mathrm{M} \Omega\end{array}$ & $\begin{array}{c}\text { Conductivity } \\
\left(\mathrm{S} \mathrm{cm}^{-1}\right)\end{array}$ \\
\hline & \multicolumn{5}{c}{ PPy } \\
Wire 1 & 16.5 & 560 & 0.76 & $0.88 \pm 0.16$ \\
Wire 2 & 16.2 & 560 & 1.41 & $0.47 \pm 0.08$ \\
Wire 3 & 16.5 & 560 & 2.49 & $0.27 \pm 0.05$ \\
& & & & \\
Wire 1 & 25.5 & 340 & 0.35 & $8.0 \pm 2.4$ \\
Wire 2 & 27.9 & 340 & 0.49 & $6.3 \pm 1.8$ \\
Wire 3 & 28.9 & 340 & 0.44 & $7.2 \pm 2.1$ \\
Wire 4 & 26.3 & 340 & 0.36 & $8.0 \pm 2.4$ \\
Wire 5 & 25.5 & 340 & 0.35 & $8.0 \pm 2.4$ \\
Wire 6 & 27.3 & 340 & 0.36 & $8.3 \pm 2.4$ \\
\hline \hline
\end{tabular}




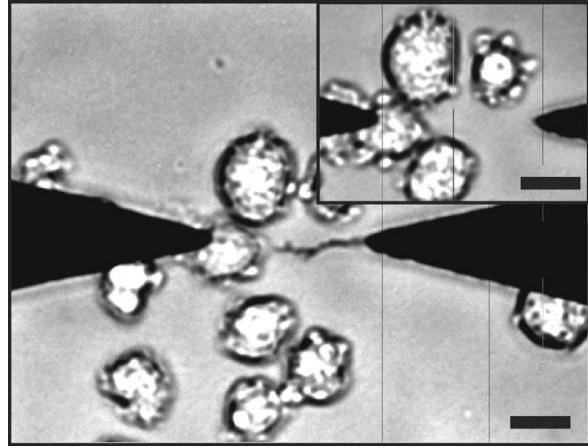

FIG. 4. A PEDOT wire grown up to a live Dictyostelium cell in the electrode gap. Inset: the initial configuration of cells. The scale bars denote $10 \mu \mathrm{m}$.

the mean. This value agrees with the previously reported conductivity value of $0.5 \mathrm{~S} \mathrm{~cm}^{-1}$ for PPy nanowires. ${ }^{21}$ The conductivities for the individual PEDOT wires were calculated to give an average value of $7.6 \pm 0.8 \mathrm{~S} \mathrm{~cm}^{-1}$ that falls within the established $0.6-10 \mathrm{~S} \mathrm{~cm}^{-1}$ range for PEDOT nanowires. ${ }^{10,22}$ Thus, the DENA technique may be used for the single-step fabrication of polymeric electrode-wireelectrode assemblies with conductances that are comparable to those produced by other approaches.

Extending this methodology to the fabrication of electrode-wire-target assemblies would provide an innovative way to deliver electrical stimuli to microscopic samples positioned in the interelectrode gap - a capability of potential electrophysiological importance. ${ }^{23}$ A critical issue in fabricating such assemblies is whether a dielectric target (such as a cell) perturbs tip-to-tip growth. The inset in Fig. 4 depicts an initial configuration of Dictyostelium cells that are foraging on the electrode array surface. One cell lies in the $30 \mu \mathrm{m}$ wide electrode gap. The phosphate buffer needed to maintain the cells contains $10 \mathrm{mM}$ EDOT and $20 \mathrm{mM}$ PSS. ${ }^{7}$ The application of a $\pm 3 \mathrm{~V}, 100 \mathrm{kHz}$ signal to the right electrode induces PEDOT wire growth. The real-time movie (Ref. 16) shows that the wire grows along the line connecting the opposing electrode tips, as in the cell-free case presented above. By decreasing the voltage-amplitude as the tip approached the cell, the wire was grown to within $1.5 \mu \mathrm{m}$ of the cell, as shown in Fig. 4. Hence, directional growth is still possible even with a dielectric cell in the interelectrode gap.

This investigation has extended the DENA-technique to the template-free growth of conducting PPy and PEDOT wires along predictable growth paths. This accomplishment enables the fabrication electrode-wire-electrode assemblies that are needed for basic transport studies ${ }^{2,3}$ and sensor applications. ${ }^{4,5}$ This methodology also enables the growth of polymeric wires from on-chip electrodes up to biological cells that are positioned in the interelectrode gap. The resulting electrode-wire-target assemblies may be used to route electrical signals to selected cells for voltage-gated signaling studies. Further work on this application is underway.

This work was supported by grants from the National Science Foundation (research: Grant Nos. PHY-646966 and EPS-0447262; instrumentation: Grant Nos. DBI-0520678 and EAR-0722410) and the National Institutes of Health (Grant No. R15 GM073698-01).

${ }^{1}$ C. K. Chiang, C. R. Fincher, Y. W. Park, A. J. Heeger, H. Shirakawa, E. J. Louis, S. C. Gau, and A. G. MacDiarmid, Phys. Rev. Lett. 39, 1098 (1977).

${ }^{2}$ A. N. Aleshin, H. J. Lee, Y. W. Park, and K. Akagi, Phys. Rev. Lett. 93, 196601 (2004).

${ }^{3}$ A. Rahman and M. K. Sanyal, Phys. Rev. B 76, 045110 (2007).

${ }^{4}$ N. T. Kemp, D. McGrouther, J. W. Cochrane, and R. Newbury, Adv. Mater. (Weinheim, Ger.) 19, 2634 (2007).

${ }^{5}$ Y. Fan, X. Chen, A. D. Trigg, C. Tung, J. Kong, and Z. Gao, J. Am. Chem. Soc. 129, 5437 (2007).

${ }^{6}$ S. M. Richardson-Burns, J. L. Hendricks, and D. C. Martin, J. Neural Eng. 4, L6 (2007).

${ }^{7}$ S. M. Richardson-Burns, J. L. Hendricks, B. Foster, L. K. Povlich, D. H. Kim, and D. C. Martin, Biomaterials 28, 1539 (2007).

${ }^{8}$ K. Ramanathan, M. A. Bangar, M. Yun, W. Chen, N. V. Myung, and A. Mulchandani, J. Am. Chem. Soc. 127, 496 (2005).

${ }^{9}$ M. Woodson and J. Liu, J. Am. Chem. Soc. 128, 3760 (2006).

${ }^{10}$ A. Das, C. H. Lei, M. Elliot, J. E. Macdonald, and M. L. Turner, Org. Electron. 7, 181 (2006).

${ }^{11}$ A. Noy, A. E. Miller, J. E. Klare, B. L. Weeks, B. W. Woods, and J. J. DeYoreo, Nano Lett. 2, 109 (2002).

${ }^{12}$ I. Talukdar, B. Ozturk, T. D. Mishima, and B. N. Flanders, Appl. Phys. Lett. 88, 221907 (2006).

${ }^{13}$ B. Ozturk, T. Mishima, D. R. Grischkowsky, and B. N. Flanders, Nanotechnology 18, 175707 (2007).

${ }^{14}$ B. Ozturk, I. Talukdar, and B. N. Flanders, Nanotechnology 18, 365302 (2007).

${ }^{15}$ B. Ozturk, C. Blackledge, D. R. Grischkowsky, and B. N. Flanders, Appl. Phys. Lett. 88, 073108 (2006).

${ }^{16}$ See EPAPS Document No. E-APPLAB-94-065903 for a real-time movie of the PEDOT wire growth process and wire growth up to a live cell. For more information on EPAPS, see http://www.aip.org/pubservs/epaps.html.

${ }^{17}$ A. F. Diaz, J. I. Castillo, J. A. Logan, and W. Y. Lee, J. Electroanal. Chem. Interfacial Electrochem. 129, 115 (1981).

${ }^{18}$ J. Mikat, I. Orgzall, and H. D. Hocheimer, Phys. Rev. B 65, 174202 (2002)

${ }^{19}$ J. P. McDonald, J. L. Hendricks, V. R. Mistry, D. C. Martin, and S. M. Yalisove, J. Appl. Phys. 102, 013107 (2007).

${ }^{20}$ S. Sakamoto, M. Okumura, Z. Zhao, and Y. Furakawa, Chem. Phys. Lett. 412, 395 (2005).

${ }^{21}$ S. S. Shiratori, S. Mori, and K. Ikezaki, Sens. Actuators B 49, 30 (1998).

${ }^{22}$ S. Samitsu, T. Shimomura, K. Ito, M. Fujimori, S. Heike, and T. Hashizume, Appl. Phys. Lett. 86, 233103 (2005).

${ }^{23}$ F. Patolsky, B. P. Timko, G. Yu, Y. Fang, A. B. Greytak, G. Zhang, and C. M. Lieber, Science 313, 1100 (2006). 